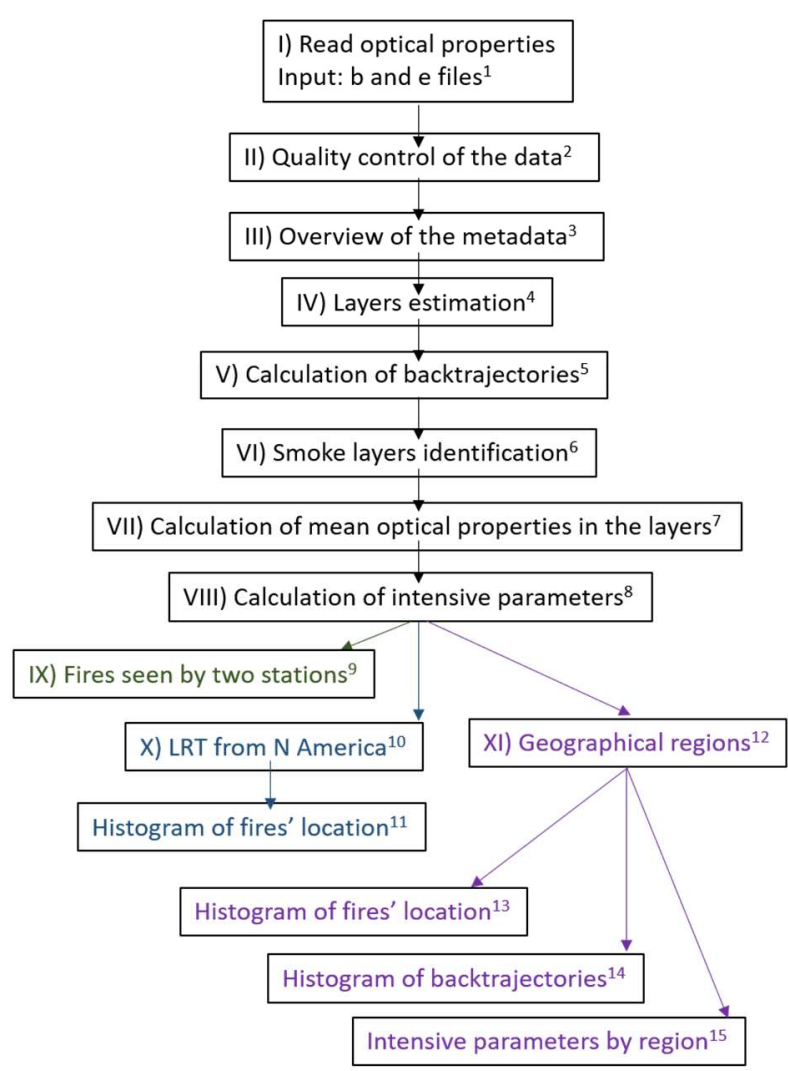

13589 input files

2 EARLINET/Forest Fire files checked following EARLINET criteria; additional inhouse checks

${ }^{3}$ e.g. detection mode, evaluation method, raw resolution

4 algorithm developed; non-accurate estimates were manually corrected; 1901 layers calculated for 960 time stamps

${ }^{5}$ run Hysplit, 10 days backwards; use GDASO $.5^{\circ}$ ( 23 cases on GDAS1 ${ }^{\circ}$ )

${ }^{6}$ use Hysplit backtrajectory and fires emissions (FIRMS); 1053 layers for 677 time stamps were considered as having BB origin

7 the average in the layer is calculated if at least $90 \%$ data is available; the averages for which SNR<2 were dismissed; there were 1050 layers for 676 time stamps for which at least one average optical property was calculated. Fig. S3.

${ }^{8}$ Values outside the range of acceptable values are dismissed. Remaining IPS are considered further (795 layers, 526 time stamps). Fig. S5.

9 based on backtrajectories, the common fires (smoke measured by two stations) were identified; compares IPs; measured smoke can be of "single fire" or "mixed fires". See Figs. 2-3.

10 based on backtrajectories, the measured smoke can be "pure N America" or "mixed" (N America + local); smoke was measured by single station or several stations; e.g. smoke measured by three stations Fig. 4, Table 2; statistics over LRT from N America (Fig. 5 and Table 3).

11 by station

12 region: SE ("atz","ino","pot","the"), NE ("cog","mas","waw"), SW

("brc","evo","gra"), CE ("cbw","lei","hpb")

13 by station; see Figs. 6-9.

14 main circulation pattern revealed for some regions.

${ }_{15}$ IPs are classified based on smoke' continental origin (Europe, N America, Asia, Africa or a mixture of them). Mean values and scatter plots are considered. Figs. 10-12, S7, Table 4, S1.

Figure S1. Methodology diagram. 
a)

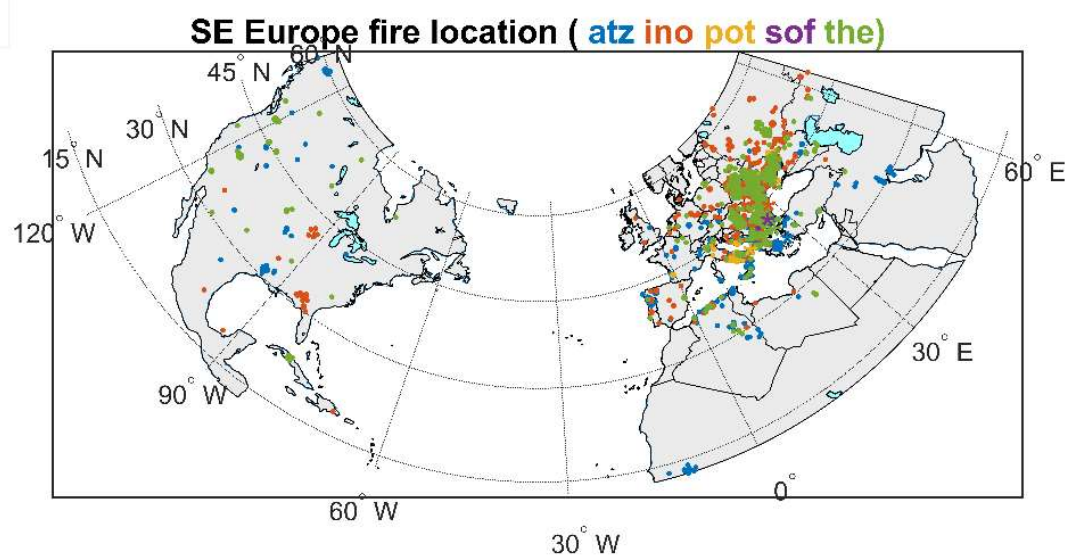

b) Histogram of fires location by station

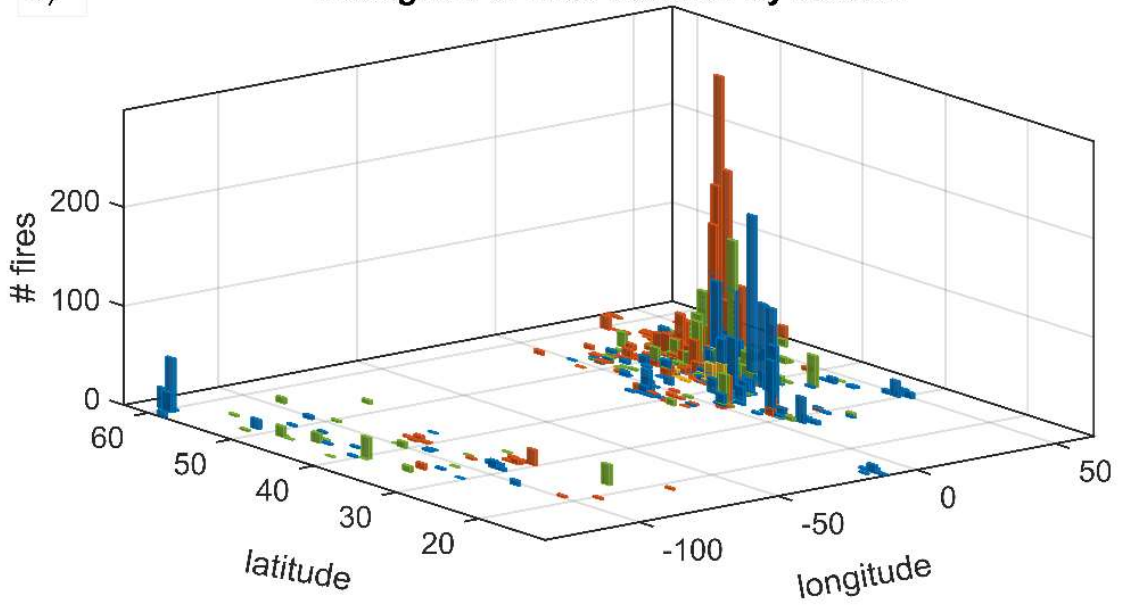

Figure S2.1. South-East Europe region formed by stations Athens (“atz"), Bucharest ("ino"), Potenza ("pot"), Sofia ("sof") and Thessaloniki ("the"). a) location of the fires detected by each station. Note that due to overlap some fires are hidden. b) histogram 5 of the fires detected by each station. 
a)
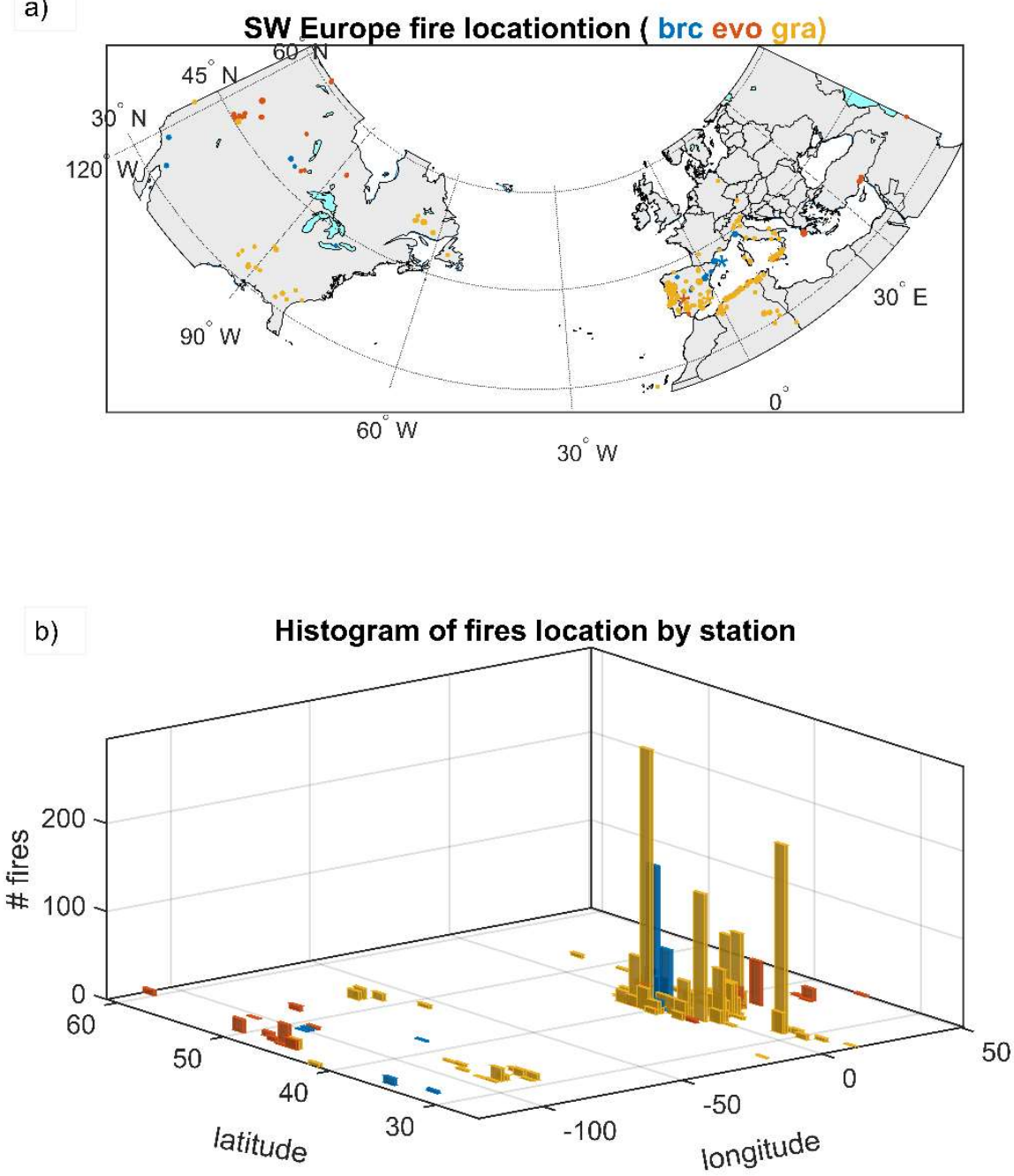

Figure S2.2. South-West Europe region formed by stations Barcelona ("brc"), Evora ("evo") and Granada ("gra"). a) location of the fires detected by each station. Note that due to overlap some fires are hidden. b) histogram of the fires detected by each station. 

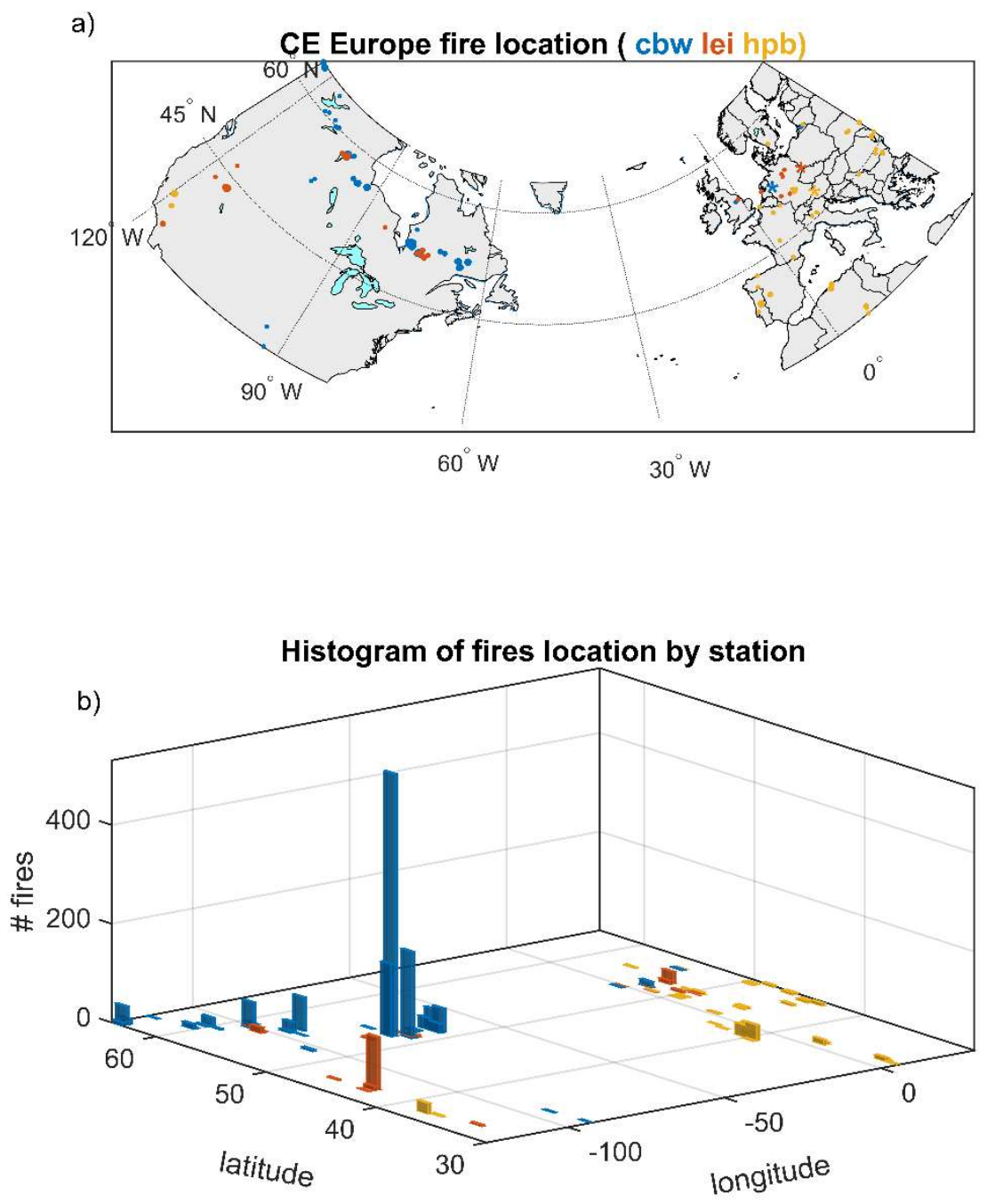

Figure S2.3. Central Europe region formed by stations Cabauw ("cbw"), Leipzig ("lei") and Hohenpeißenberg ("hpb"). a) location of the fires detected by each station. Note that due to overlap some fires are hidden. b) histogram of the fires detected by each station. 
a)

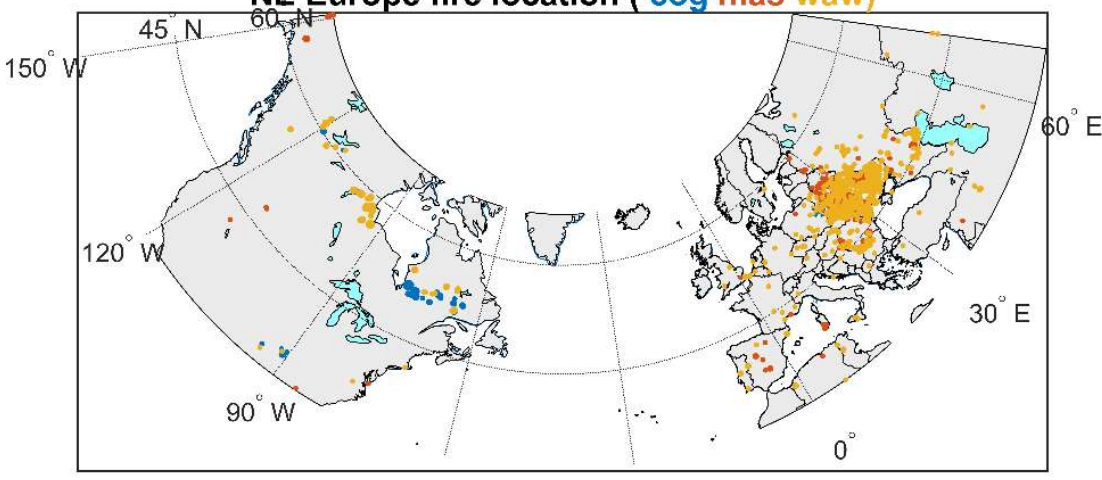

$60^{\circ} \mathrm{W}$

$30^{\circ} \mathrm{W}$

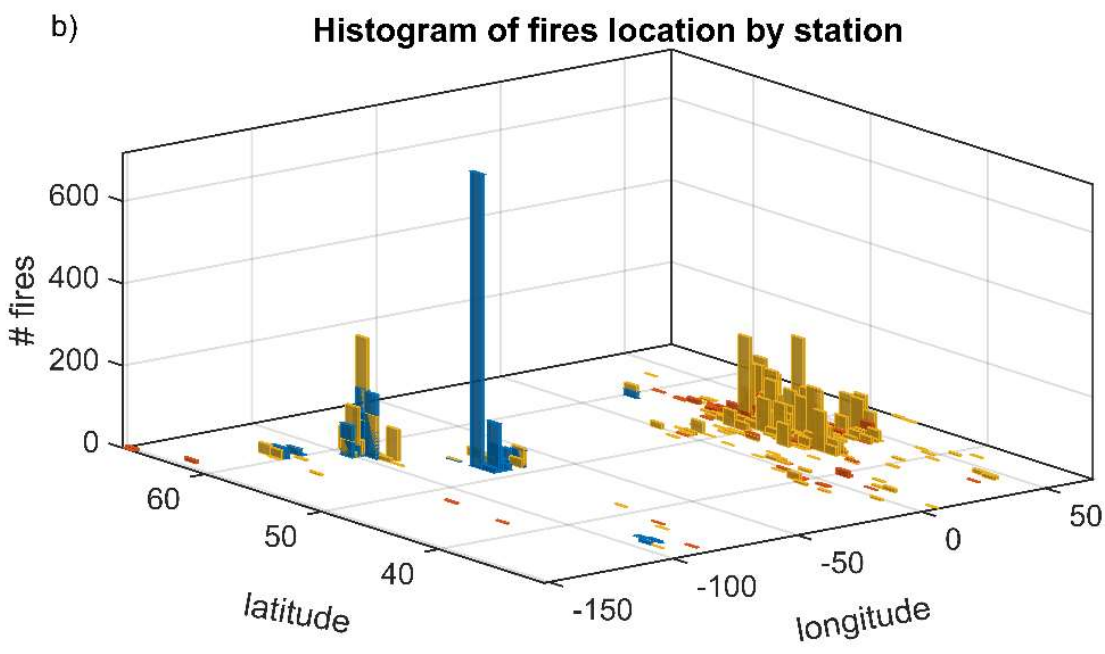

Figure S2.4. North-East Europe region formed by stations Belsk ("cog"), Minsk ("mas") and Warsaw ("waw"). a) location of the fires detected by each station. Note that due to overlap some fires are hidden. b) histogram of the fires detected by each station. 


\section{S3. Histogram of the backtrajectories}

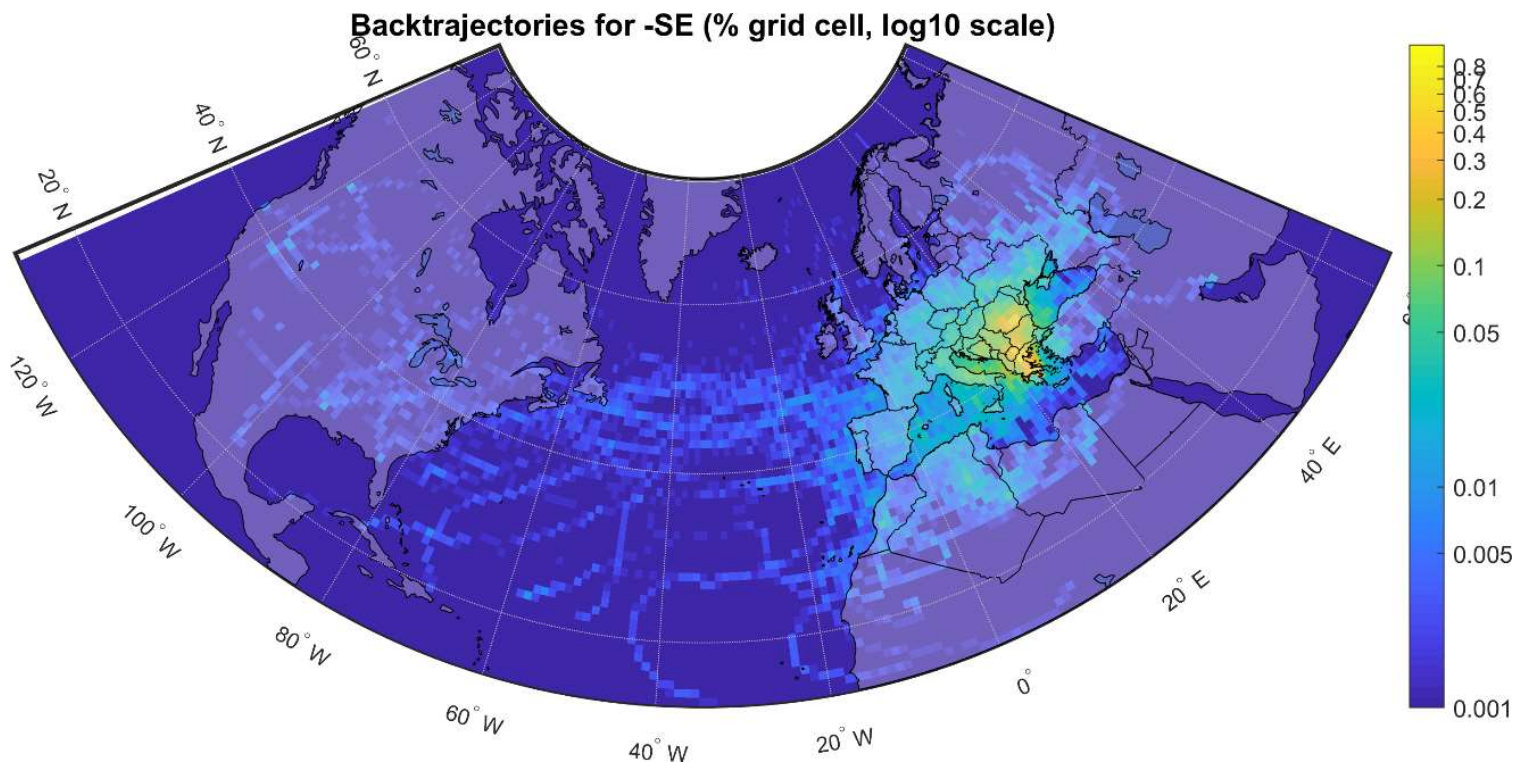

Figure S3.1. Histogram of the backtrajectories for SE region. '\% grid cell' represent the percentage of the number of backtrajectories in a grid with respect to the total number of the backtrajectories.

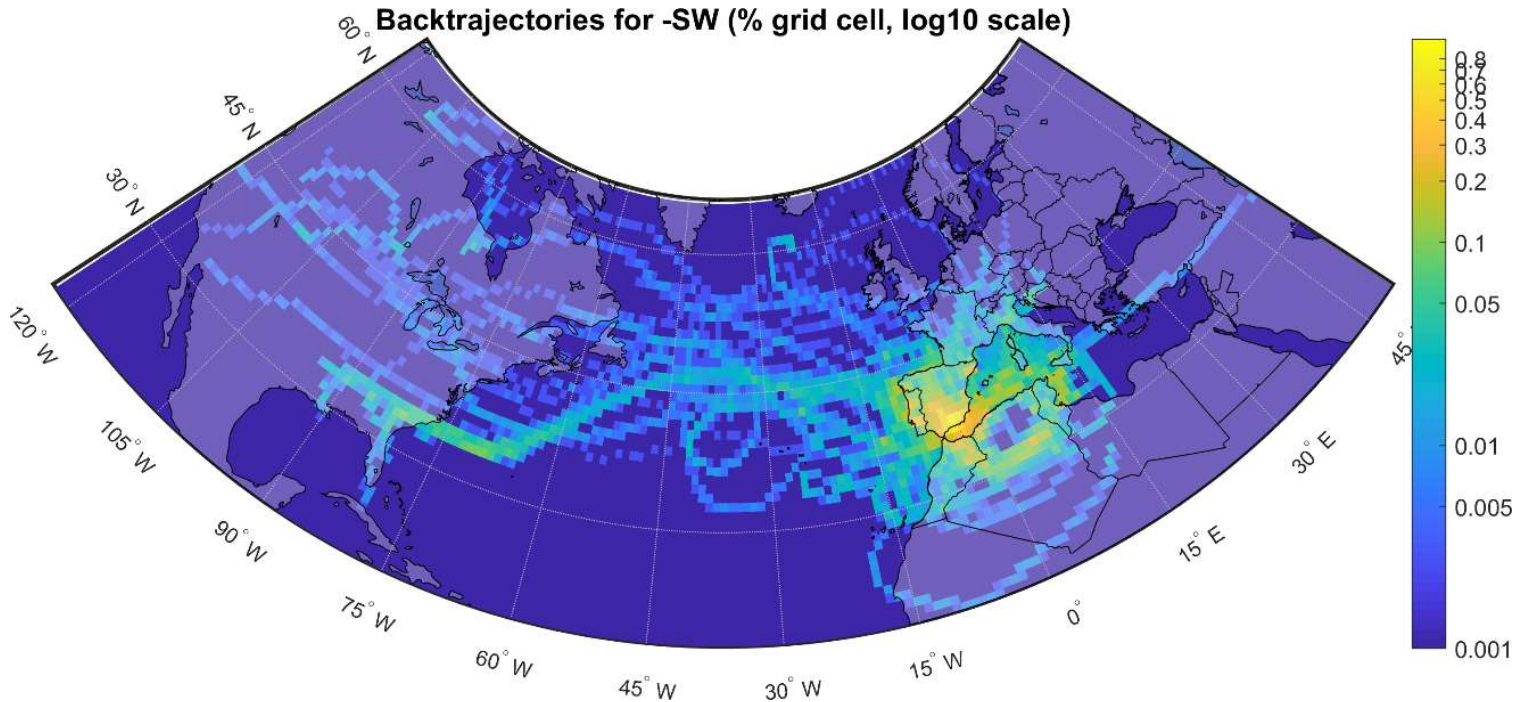

Figure S3.2. Histogram of the backtrajectories for SW region. 


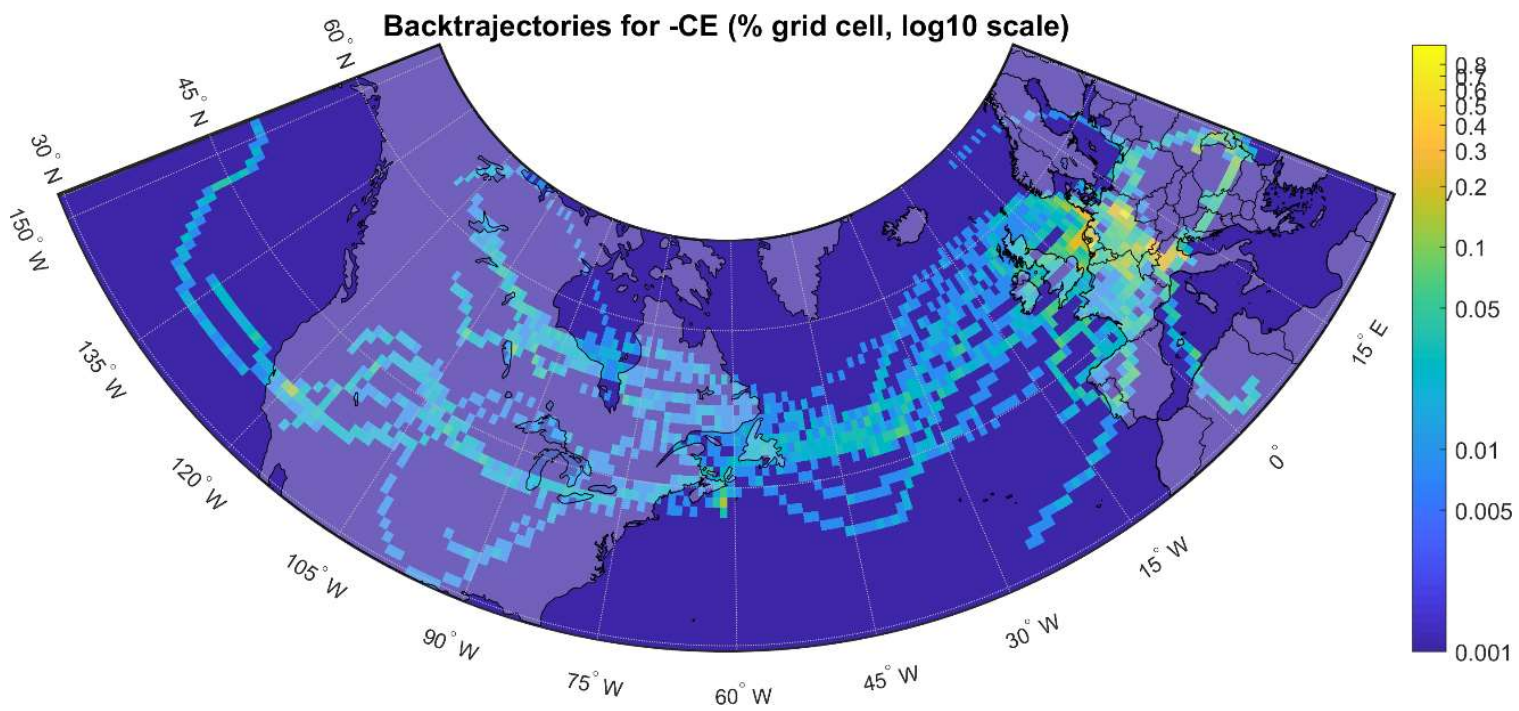

Figure S3.3. Histogram of the backtrajectories for $\mathrm{CE}$ region.

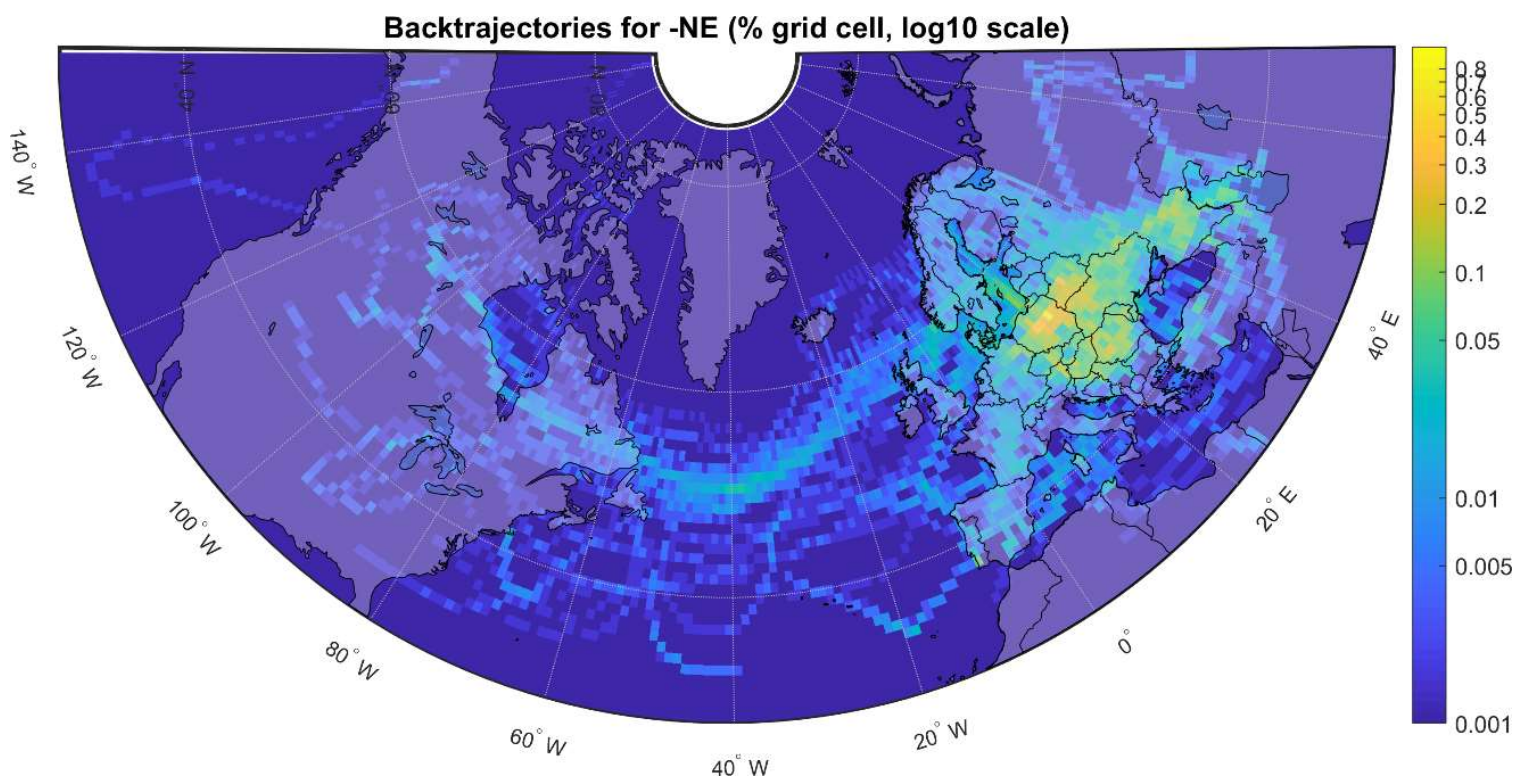

Figure S3.4. Histogram of the backtrajectories for NE region. 

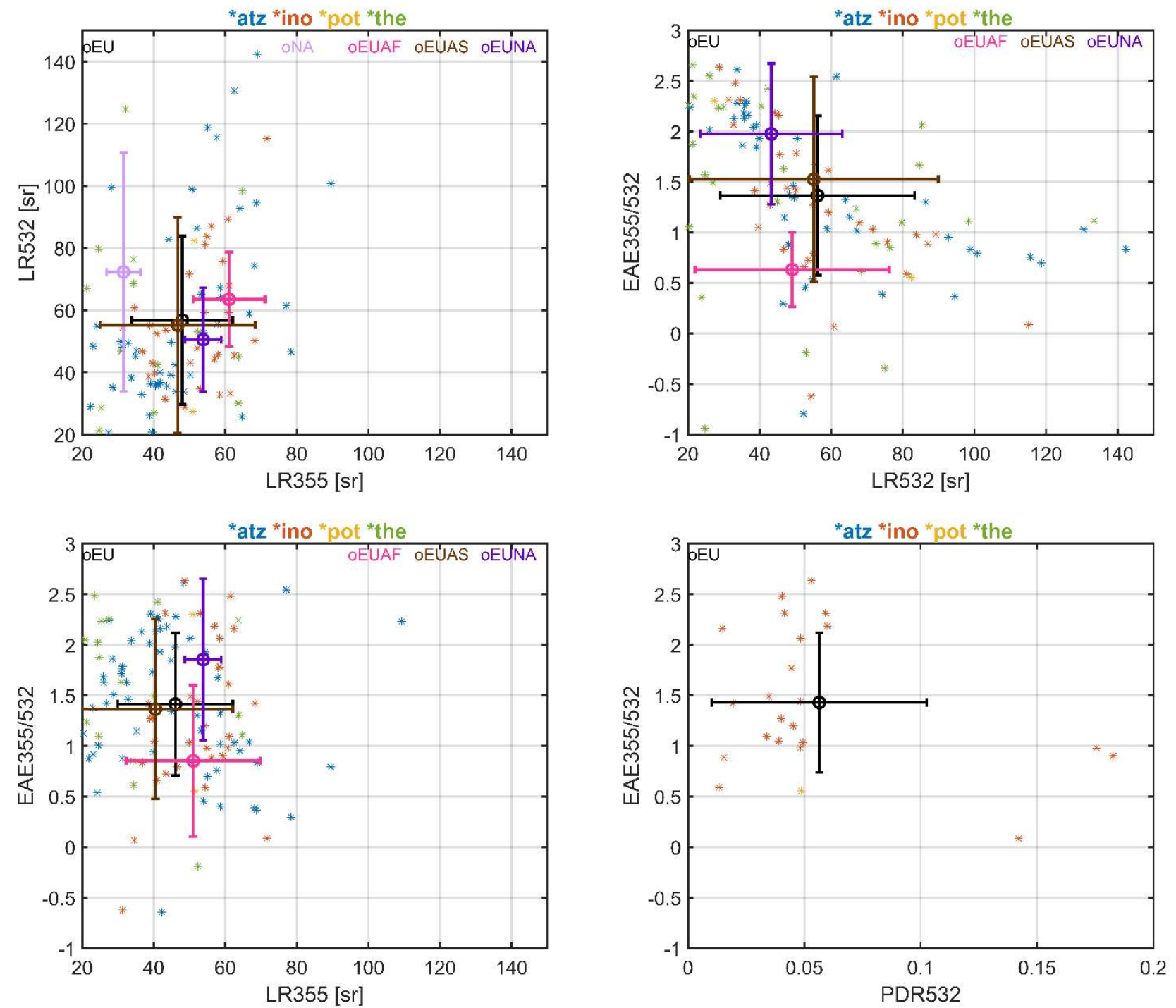

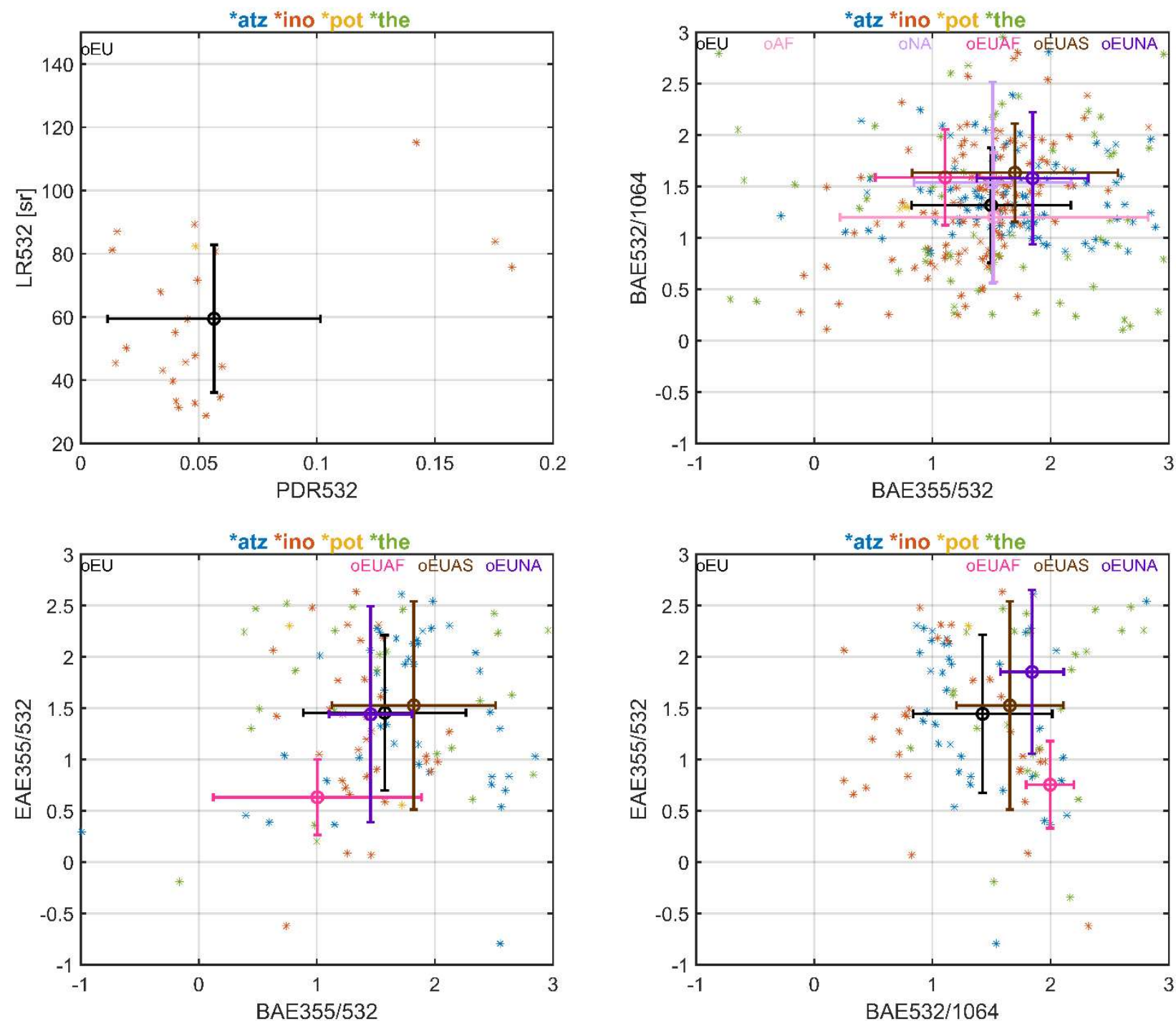

Figure S4.1. Scatter plots between various two intensive parameters for South-East (SE) region. The colour code of the asterisks is station related (as labelled in the title). The colour code for the mean values (shown by circles) and their STD values (shown by error bars) are related to the smoke source origin (stated as text on the plots). Thus, we have the following source origins: Europe (EU black), Africa (AF - light pink), Asia (AS - light brown), North America (NA - light magenta), Europe - Africa (EUAF - pink), Europe - Asia (EUAS - brown) and Europe - North America (EUNA - magenta). 

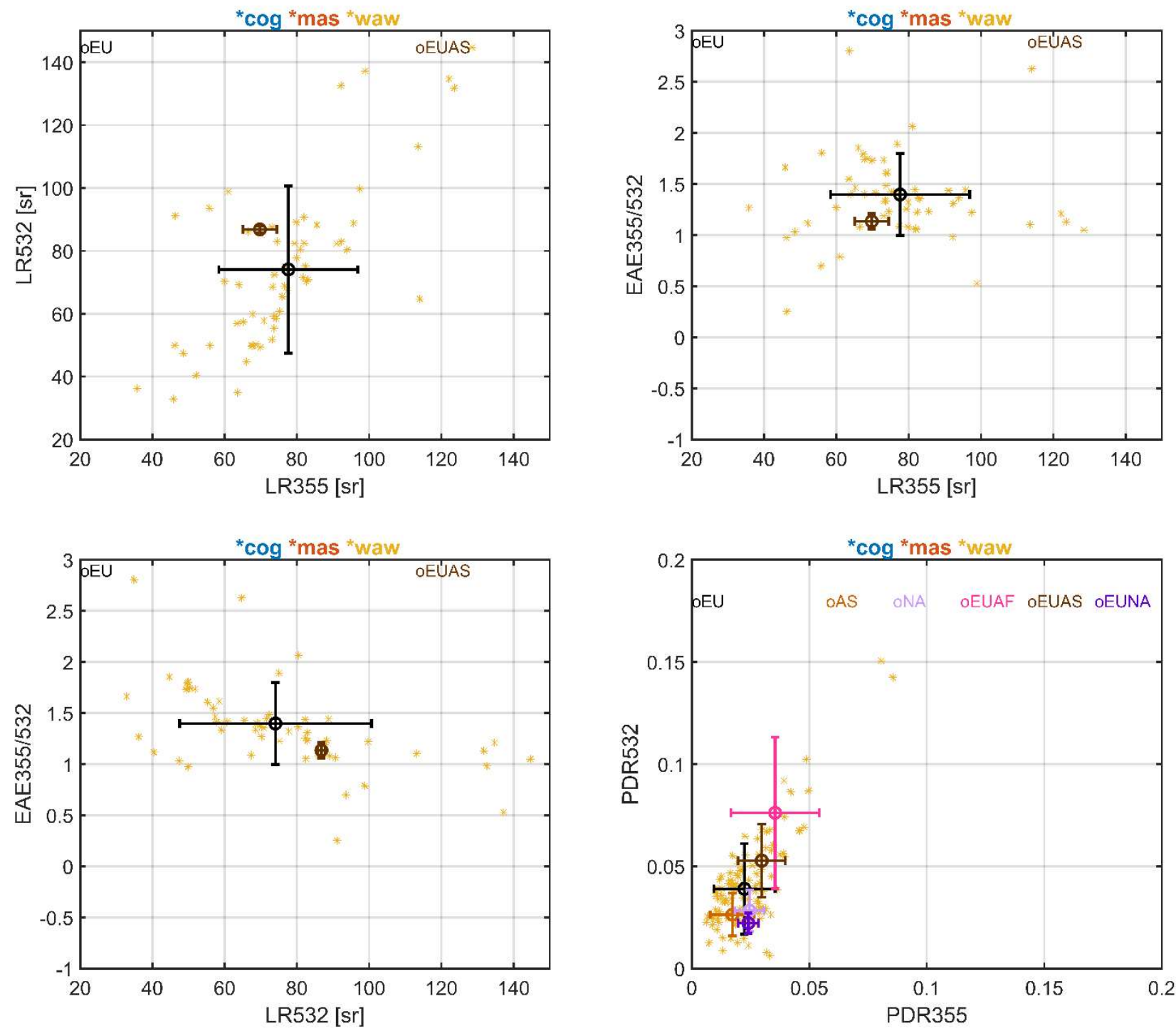

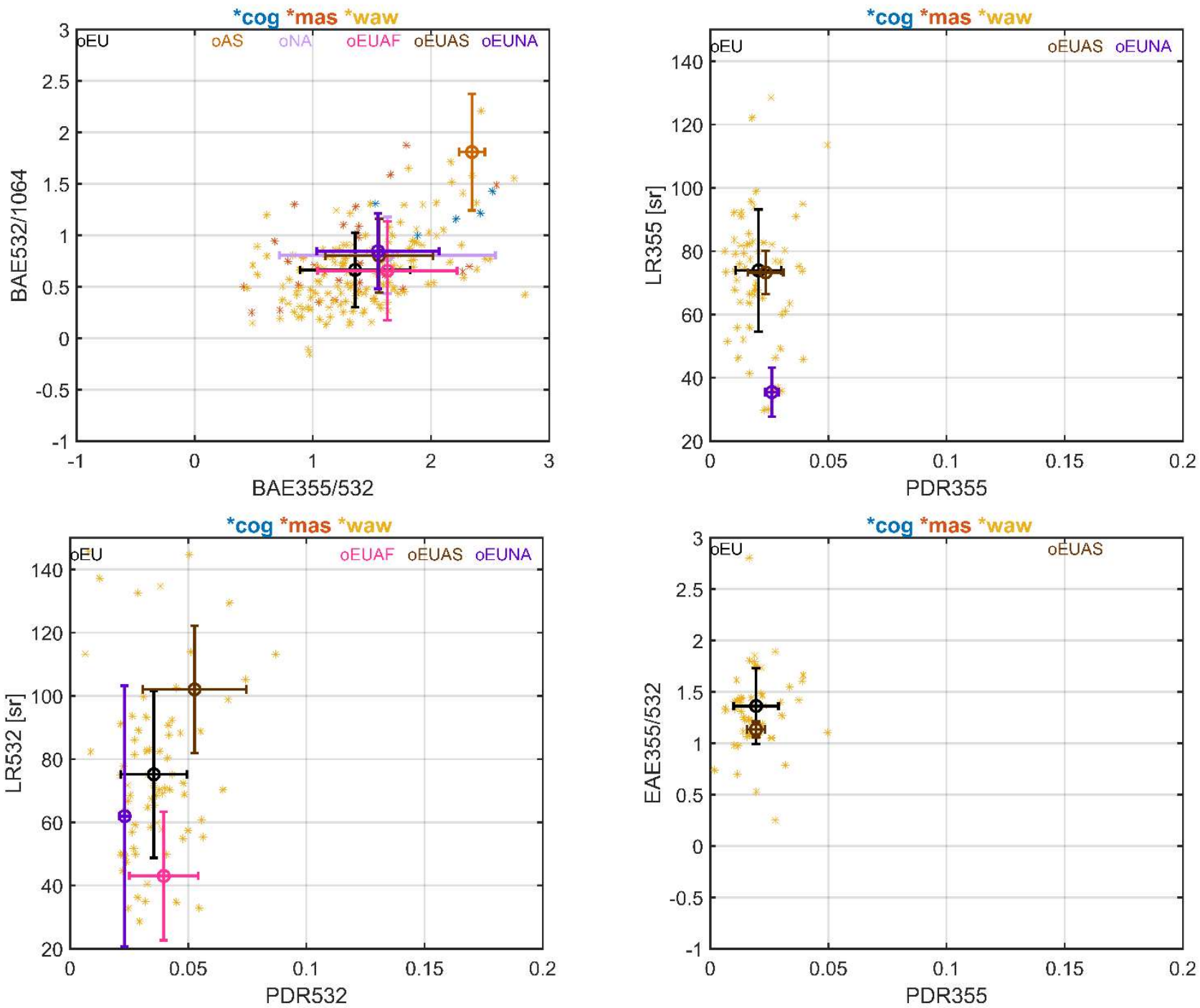

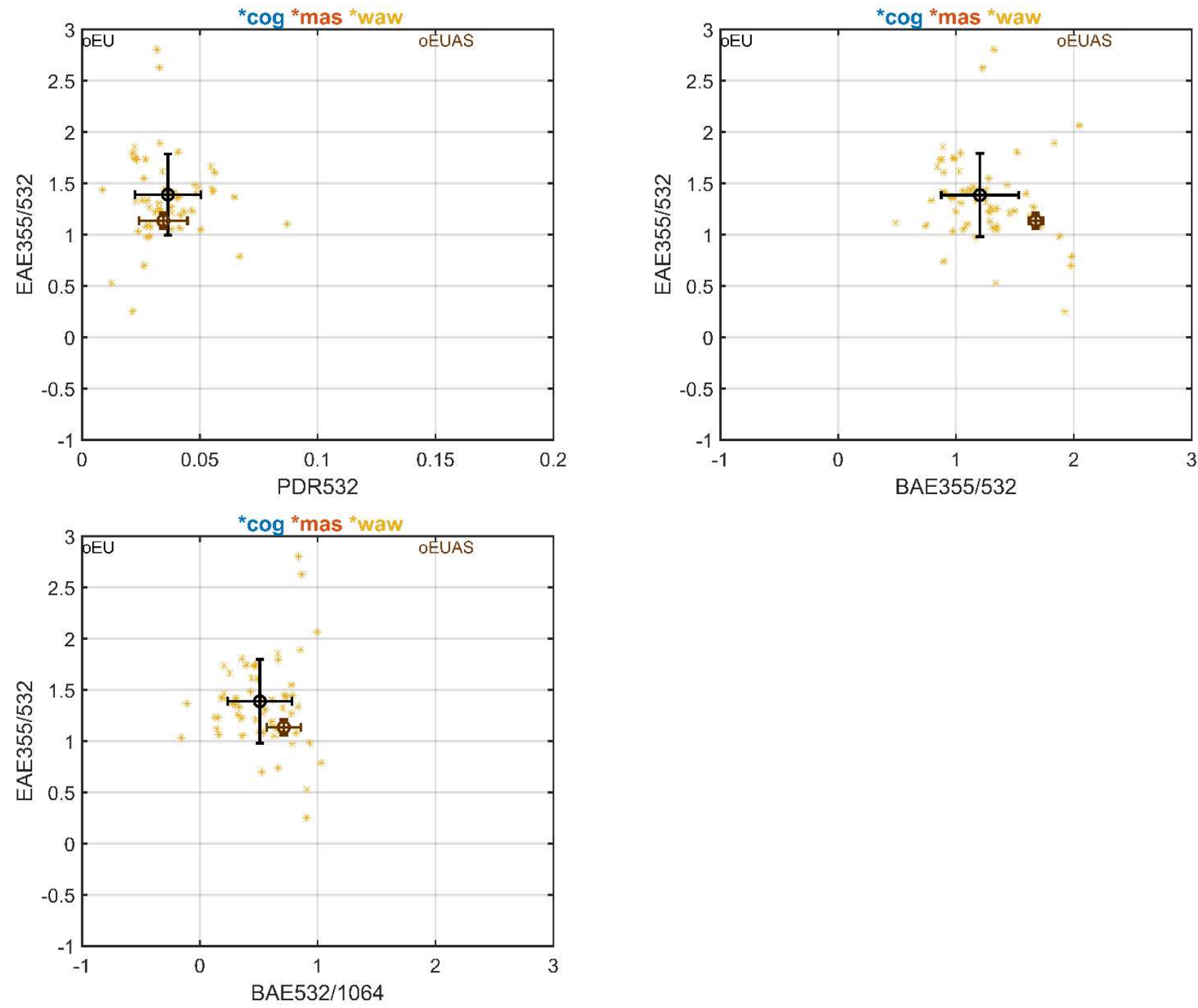

Figure S4.2. Scatter plots between various two intensive parameters for North-East (NE) region. The colour code of the asterisks is station related (as labelled in the title). The colour code for the mean values (shown by circles) and their STD values (shown by error 5 bars) are related to the smoke source origin (stated as text on the plots). Thus, we have the following source origins: Europe (EU black), Africa (AF - light pink), Asia (AS - light brown), North America (NA - light magenta), Europe - Africa (EUAF - pink), Europe - Asia (EUAS - brown) and Europe - North America (EUNA - magenta). 

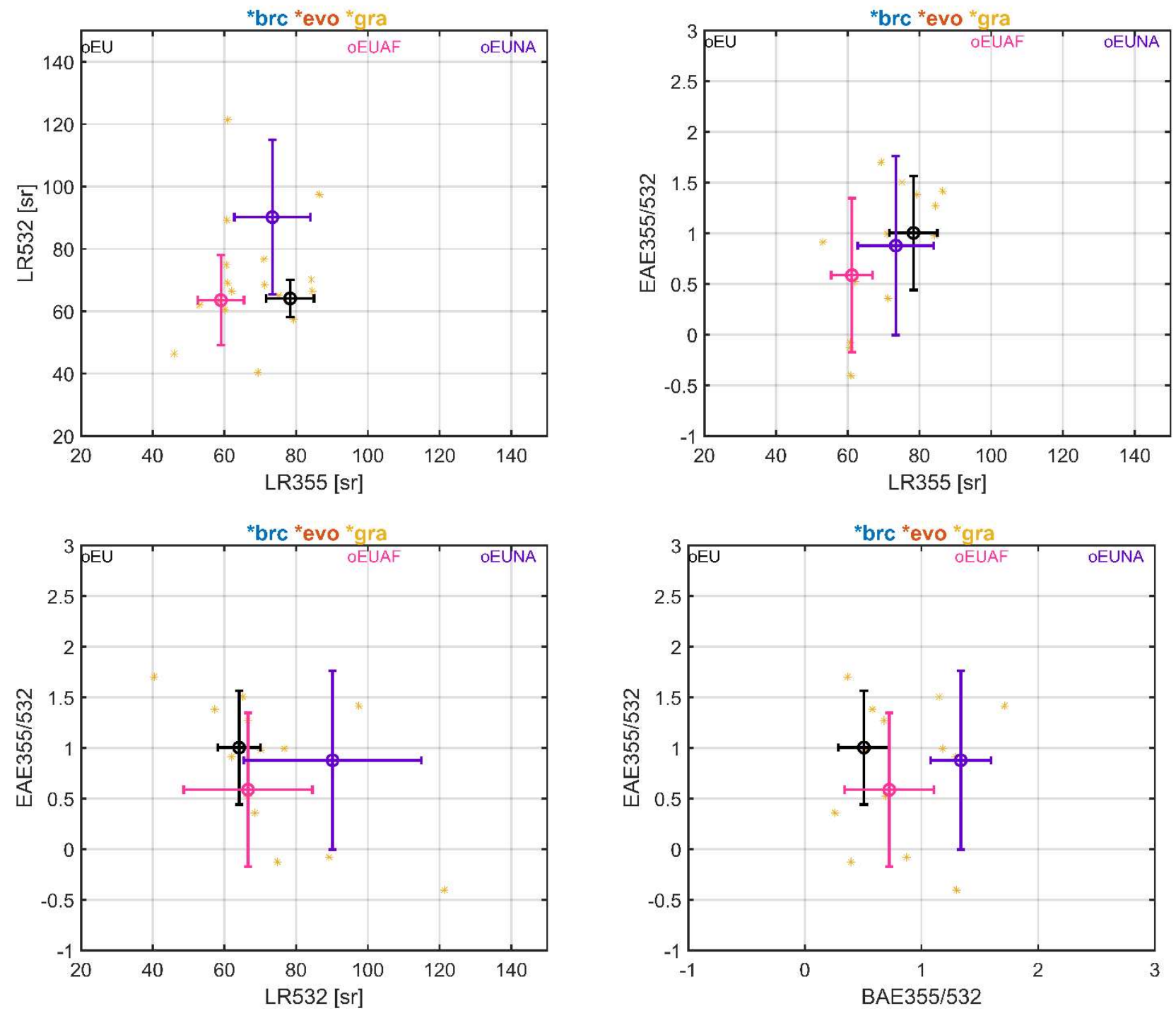

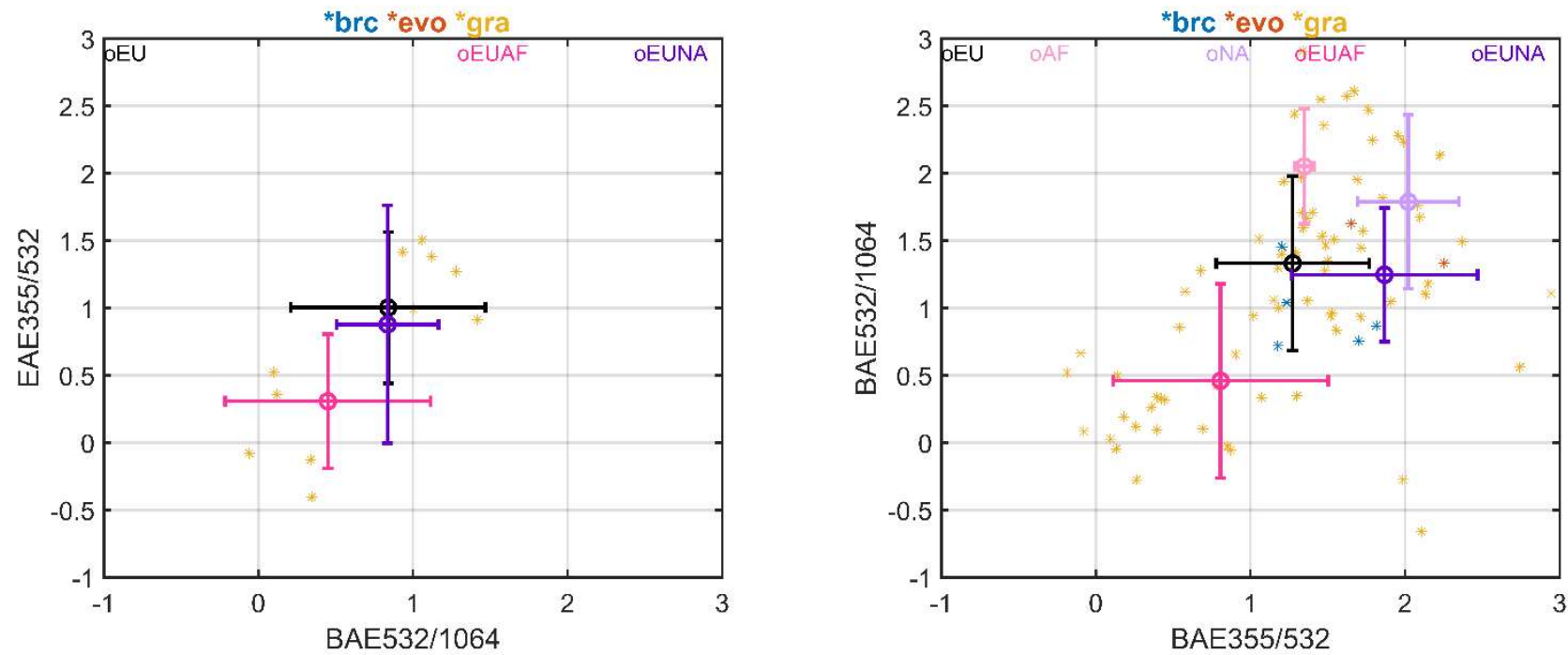

Figure S4.3. Scatter plots between various two intensive parameters for South-West (SE) region. The colour code of the asterisks is station related (as labelled in the title). The colour code for the mean values (shown by circles) and their STD values (shown by error bars) are related to the smoke source origin (stated as text on the plots). Thus, we have the following source origins: Europe (EU -

5 black), Africa (AF - light pink), Asia (AS - light brown), North America (NA - light magenta), Europe - Africa (EUAF - pink), Europe - Asia (EUAS - brown) and Europe - North America (EUNA - magenta). 

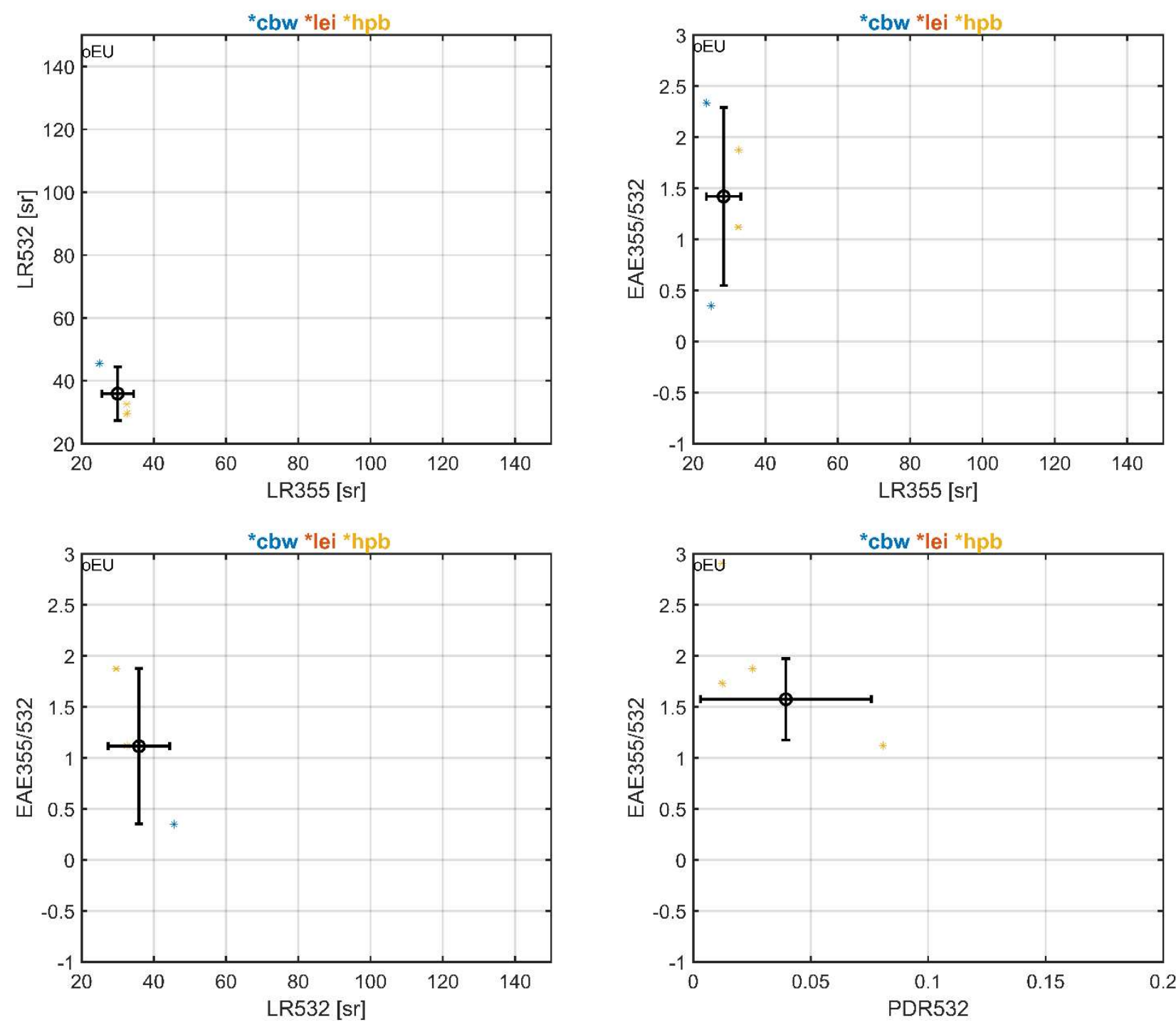

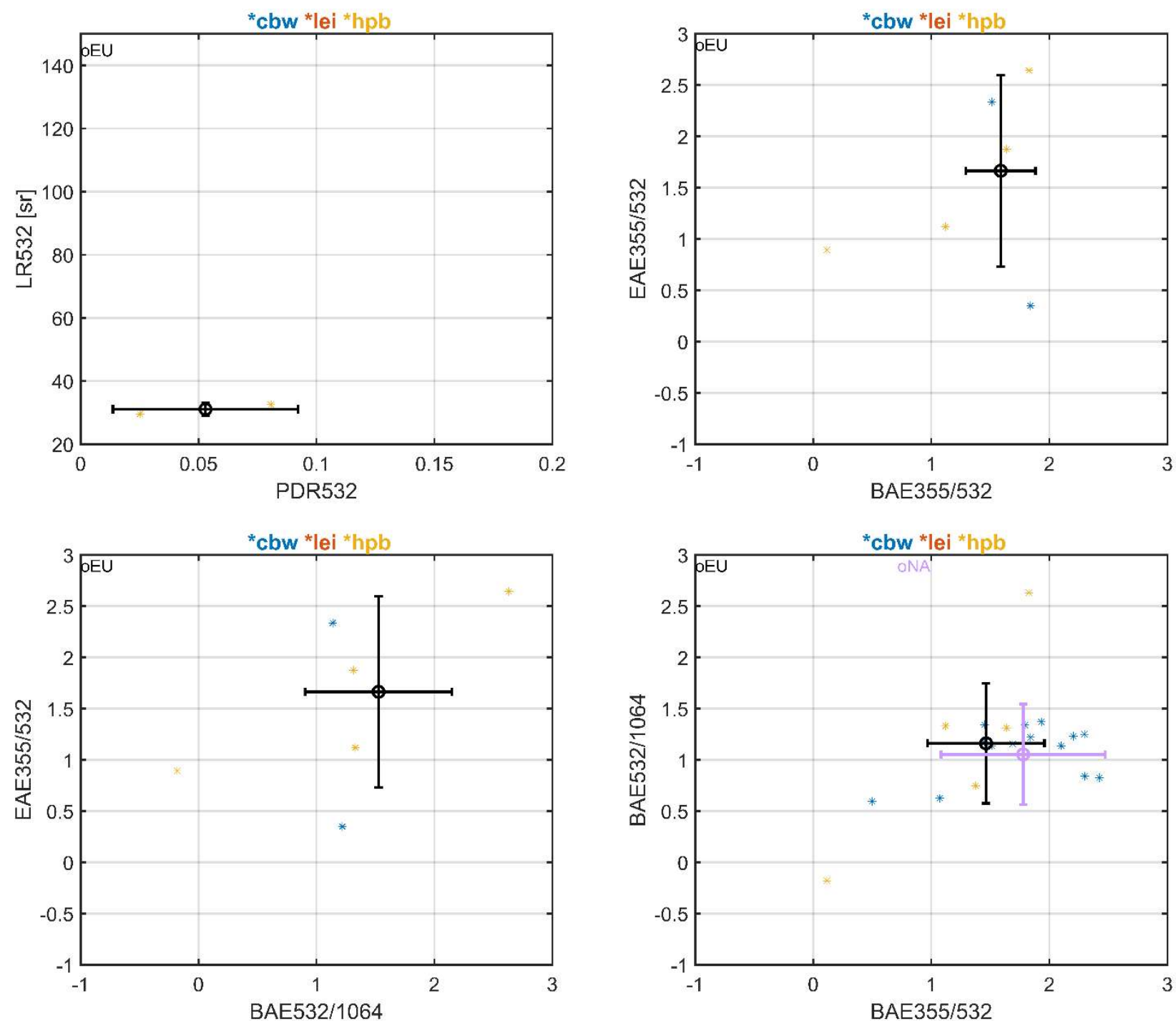

Figure S4.4. Scatter plots between various two intensive parameters for Central (CE) region. The colour code of the asterisks is station related (as labelled in the title). The colour code for the mean values (shown by circles) and their STD values (shown by error bars) are related to the smoke source origin (stated as text on the plots). Thus, we have the following source origins: Europe (EU black), Africa (AF - light pink), Asia (AS - light brown), North America (NA - light magenta), Europe - Africa (EUAF - pink), Europe - Asia (EUAS - brown) and Europe - North America (EUNA - magenta). 